\title{
Conjunctive and Disjunctive Limits: Abstract Logics and Modal Operators
}

\author{
Alexandre Costa-Leite \\ University of Brasilia \\ Campus Darcy Ribeiro - ICC - Ala Norte \\ CEP 70910-900 \\ Brasilia DF Brazil \\ e-mail:costaleite@unb.br
}

Edelcio G. de Souza

University of Sao Paulo

Rua Luciano Gualberto, 315

CEP 05508-010

Sao Paulo Brazil

e-mail: edelcio.souza@usp.br

\begin{abstract}
:
Departing from basic concepts in abstract logics, this paper introduces two concepts: conjunctive and disjunctive limits. These notions are used to formalize levels of modal operators.

Keywords: abstract logic, conjunctive limit, disjunctive limit, modal logic, Jan Woleński.
\end{abstract}

\section{Introduction}

There are families of concepts organized by some order and some kind of hierarchy. This phenomenon occurs in distinct areas of logic: sequences of sentences can be systematized to highlight the most essential element in the sequence (the sovereign object in the hierarchy). In this article, we use the concept of limit of a given sequence to redefine the notions of conjunctive limit and disjunctive limit in the universe of abstract logic. ${ }^{1}$ By means of this strategy, we can formulate specific standards of logical possibility as well logical necessity pointing out that the same procedure could be extended to a great variety of sequences of objects (with very different natures, indeed).

We start introducing main useful concepts from abstract logic and, then, in section 3, we present some notions such as those of conjunctive limit, disjunctive limit as well the concepts of 
conjunctive logic and disjunctive logic. In section 4, some of these ideas are applied to reason about levels of modal operators.

\section{Concepts in Abstract Logics}

We establish several basic preliminary and standard concepts in the realm of general abstract logic following initial ideas developed by Alfred Tarski in [9] and [10]. ${ }^{2}$ This approach to logical consequence sounds awesome and very useful allowing us to be well oriented through the incredible plurality of rationalities displayed by the great variety of logical systems.

An abstract logic is a pair $\mathrm{L}=\left(\mathrm{S}, \mathrm{Cn}_{\mathrm{L}}\right)$ such that $\mathrm{S}$ is a non-empty set and $\mathrm{Cn}_{\mathrm{L}}$ is a map

$$
\mathrm{Cn}_{\mathrm{L}}: \wp(\mathrm{S}) \rightarrow \wp(\mathrm{S})
$$

in the power set of $\mathrm{S}$. The operator $\mathrm{Cn}_{\mathrm{L}}$ should satisfy: ${ }^{3}$

i. Inclusion: $\mathrm{A} \subseteq \mathrm{Cn}(\mathrm{A})$.

ii. Idempotency: $\mathrm{Cn}(\mathrm{Cn}(\mathrm{A}))=\mathrm{Cn}(\mathrm{A})$.

iii. Monotonicity: $\mathrm{Cn}(\mathrm{A}) \subseteq \mathrm{Cn}(\mathrm{A} \cup \mathrm{B})$.

We call $\mathrm{S}$ the domain or universe of $\mathrm{L}$ and $\mathrm{Cn}_{\mathrm{L}}$ is its consequence operator. Elements of $\mathrm{S}$ are called sentences and, therefore, we are concerned with logical consequence defined for sentences. ${ }^{4}$

As it is well-known, consequence operators are connected with consequence relations by means of a very natural relationship. Given an abstract logic $\mathrm{L}=\left(\mathrm{S}, \mathrm{Cn} \mathrm{L}_{\mathrm{L}}\right)$, it is feasible to define a binary relation

$$
H_{\mathrm{L}} \subseteq \wp(\mathrm{S}) \times \mathrm{S}
$$

such that:

$$
\text { A } \vdash_{L} \text { a iff (if and only if) } \mathrm{a} \in \mathrm{Cn}_{\mathrm{L}}(\mathrm{A}) \text {. }
$$

We call $-_{\mathrm{L}}$ the consequence relation of L. It is easy to see that $\mathrm{H}_{\mathrm{L}}$ satisfies: $^{5}$

I. Inclusion: If $\mathrm{a} \in \mathrm{A}$, then $\mathrm{A} \vdash \mathrm{a}$.

II. Transitivity: If $\left.\mathrm{B}\right|_{\mathrm{L}}$ a and $\mathrm{A} \mid-\mathrm{b}$ for all $\mathrm{b} \in \mathrm{B}$ then $\mathrm{A} \mid-\mathrm{a}$.

III. Monotonicity: If $\mathrm{A}-\mathrm{a}$ and $\mathrm{A} \subseteq \mathrm{B}$, then $\mathrm{B} \mid-\mathrm{a}$.

I and III are immediate. For II, suppose that $\mathrm{a} \in \mathrm{Cn}(\mathrm{B})$ and $\mathrm{b} \in \mathrm{Cn}(\mathrm{A})$ for all $\mathrm{b} \in \mathrm{B}$. Thus, $\mathrm{B} \subseteq$ $\mathrm{Cn}(\mathrm{A})$. By idempotency and monotonicity, we have:

$$
\mathrm{Cn}(\mathrm{B}) \subseteq \mathrm{Cn}(\mathrm{Cn}(\mathrm{A}))=\mathrm{Cn}(\mathrm{A})
$$

Then, $\mathrm{a} \in \mathrm{Cn}(\mathrm{A})$, that is, $\mathrm{A} \mid-\mathrm{a}$.

Now, let $\mathrm{L}=\left(\mathrm{S}, \mathrm{C} \mathrm{n}_{\mathrm{L}}\right)$ be an abstract logic. We say that:

(a) $\mathrm{A} \subseteq \mathrm{S}$ is L-limited iff $\mathrm{Cn}_{\mathrm{L}}(\mathrm{A}) \neq \mathrm{S} .{ }^{6}$ Otherwise, $\mathrm{A}$ is L-unlimited;

(b) A sentence $\mathrm{c} \in \mathrm{S}$ is a 0 -sentence iff $\mathrm{Cn}_{\mathrm{L}}(\{\mathrm{c}\})$ is L-unlimited. Moreover, if $\mathrm{t} \in \mathrm{Cn}_{\mathrm{L}}(\varnothing)$ we say that $\mathrm{t}$ is a 1 -sentence.

We denote by $\mathbf{1}_{\mathrm{L}}$ and $\mathbf{0}_{\mathrm{L}}$ the set of all 1 -sentences and 0 -sentences, respectively. The four above notions are in some sense related with the traditional concepts of consistency, inconsistency, 
contradiction and tautology, respectively. Here these concepts are dressed with new clothes to be more adaptable to our purposes departing from a general perspective.

Let $\mathrm{L}=\left(\mathrm{S}, \mathrm{Cn} \mathrm{L}_{\mathrm{L}}\right)$ be an abstract logic. We can use the consequence operator $\mathrm{Cn}_{\mathrm{L}}$ in order to define a partial order in the set of sentences $S$.

If $x, y \in S$, we define:

$$
\mathrm{x} \leq \mathrm{y} \text { iff }\left.\{\mathrm{x}\}\right|_{\mathrm{L}} \mathrm{y} .
$$

It is clear that $\leq$ is reflexive and transitive. Then, $(X, \leq)$ is a partially ordered set. In this setting, we can take into account upper and lower bounds, supremum, infimum, maximal and minimal elements etc.

We use $\mathrm{Cn}_{\mathrm{L}}$ to define, for $\mathrm{x}, \mathrm{y} \in \mathrm{S}$, an equivalence relation between elements of $\mathrm{S}$ in the following way:

$$
\mathrm{x} \equiv \mathrm{y} \text { iff } \mathrm{Cn}_{\mathrm{L}}(\{\mathrm{x}\})=\mathrm{Cn}_{\mathrm{L}}(\{\mathrm{y}\}) .
$$

In this case, we have: $\{\mathrm{x}\} \vdash_{\mathrm{L}} \mathrm{y}$ and $\{\mathrm{y}\} \mathrm{-}_{\mathrm{L}} \mathrm{x}$. It is easy to see that $\equiv$ is an equivalence relation and, as usual, we have that:

$$
[\mathrm{x}]_{\equiv}=\{\mathrm{y} \in \mathrm{S}: \mathrm{x} \equiv \mathrm{y}\}
$$

Therefore, the quotient set is given by

$$
\mathrm{S} / \equiv=\left\{[\mathrm{x}]_{\equiv} \in \wp(\mathrm{S}): \mathrm{x} \in \mathrm{S}\right\}
$$

The order relation $\leq$ is transferred to the set $\mathrm{S} / \equiv^{7}$

$$
[\mathrm{x}] \leq[\mathrm{y}] \text { iff } \mathrm{x} \leq \mathrm{y} .
$$

This construction does not depend on the representatives $\mathrm{x}$ and $\mathrm{y}$.

Given an abstract logic $\mathrm{L}=(\mathrm{S}, \mathrm{Cn} \mathrm{L})$, if the sets $\mathbf{1}_{\mathrm{L}}$ and $\mathbf{0}_{\mathrm{L}}$ are not empty, then the sets $\mathbf{1}_{\mathrm{L}}$ and $\mathbf{0}_{\mathrm{L}}$ are the greatest and the lowest elements in the ordered set $\mathrm{S} / \equiv$.

Dealing with logics from this abstract viewpoint sounds very elegant and useful, especially considering the mess caused by the plurality of rationalities that one can find in the market. And, more important, this approach is essential to our next definitions.

\section{Conjunctive and Disjunctive Limits}

The original ideas of conjunctive and disjunctive limits introduced in this section appeared inside a different framework in [4]. These concepts are here reformulated in the spirit of abstract logic. From now on, it follows the main contributions of this paper.

Consider an abstract logic $\mathrm{L}=\left(\mathrm{S}, \mathrm{Cn} \mathrm{n}_{\mathrm{L}}\right)$. Let $\left(\mathrm{x}_{\mathrm{i}}\right)_{\mathrm{i} \in \omega}$ be a sequence of elements of $\mathrm{S}$. We say that $[x]_{\equiv} \in \mathrm{S} / \equiv$ is a conjunctive limit of $\left(\mathrm{x}_{\mathrm{i}}\right)_{\mathrm{i} \in \omega}$ iff there exists $\mathrm{k} \in \omega$ such that for $\mathrm{i} \geq \mathrm{k}$, we have $\{\mathrm{x}\}$ $-_{\mathrm{L}} \mathrm{x}_{\mathrm{i}}$ (or, that is the same, $\left.\mathrm{x} \leq \mathrm{x}_{\mathrm{i}}\right)$. The set of all conjunctive limits of $\left(\mathrm{x}_{\mathrm{i}}\right)_{\mathrm{i} \in \omega}$ is denoted by $\mathbf{L I M}^{\mathrm{c}}\left(\mathrm{x}_{\mathrm{i}}\right)$. Notice that if $\mathrm{c}$ is a 0 -sentence, then $[\mathrm{c}]_{\equiv}=\mathbf{0}_{\mathrm{L}}$ is a conjunctive limit of all sequences of elements of S. This allows us to talk about a formula from which all other formulas can be derived. ${ }^{8}$

The construction above can be dualized. We say that $[\mathrm{x}]_{\equiv} \in \mathrm{S} / \equiv$ is a disjunctive limit of $\left(x_{i}\right)_{i \in \omega}$ iff there exists $k \in \omega$ such that for $i \geq k$, we have $\left\{x_{i}\right\} L_{L} x$ (or, that is the same, $x_{i} \leq x$ ). The set of all disjunctive limits of $\left(x_{i}\right)_{i \in \omega}$ is denoted by $\operatorname{LIM}^{\mathrm{d}}\left(\mathrm{x}_{\mathrm{i}}\right)$. In this case, if $\mathrm{t}$ is a 1 -sentence, then $[\mathrm{t}]_{\equiv}=\mathbf{1}_{\mathrm{L}}$ is a disjunctive limit of all sequences of elements of $\mathrm{S}$. Now, this allows us to talk about a formula which is a consequence of all other formulas. 
An abstract logic $\mathrm{L}=\left(\mathrm{S}, \mathrm{Cn}_{\mathrm{L}}\right)$ is conjunctive iff for all sequence $\left(\mathrm{x}_{\mathrm{i}}\right)_{\mathrm{i} \in \omega}$ of elements of $\mathrm{S}$, the set $\mathbf{L I M}^{\mathrm{c}}\left(\mathrm{x}_{\mathrm{i}}\right)$ has the minimum. In this case, we define:

$$
\lim ^{\mathrm{c}}\left(\mathrm{x}_{\mathrm{i}}\right):=\min \left(\mathbf{L I M}^{\mathrm{c}}\left(\mathrm{x}_{\mathrm{i}}\right)\right)
$$

A $\operatorname{logic} L=\left(S, C n_{L}\right)$ is properly conjunctive iff for all sequence $\left(x_{i}\right)_{i \in \omega}$ of elements of $S$, the set $\mathbf{L I M}^{\mathbf{c}}\left(\mathrm{x}_{\mathrm{i}}\right)$ has the minimum that it is not $\mathbf{0}_{\mathrm{L}}$.

We also have an immediate dual concept: an abstract logic $\mathrm{L}=\left(\mathrm{S}, \mathrm{Cn}_{\mathrm{L}}\right)$ is disjunctive iff for all sequence $\left(\mathrm{x}_{\mathrm{i}}\right)_{\mathrm{i} \in \omega}$ of elements of $\mathrm{S}$, the set $\mathbf{L} \mathbf{I} \mathbf{M}^{\mathrm{d}}\left(\mathrm{x}_{\mathrm{i}}\right)$ has the maximum. In this case, we define:

$$
\lim ^{\mathrm{d}}\left(\mathrm{x}_{\mathrm{i}}\right):=\max \left(\mathbf{L I M}^{\mathrm{d}}\left(\mathrm{x}_{\mathrm{i}}\right)\right) .
$$

A $\operatorname{logic} L=\left(S, C n_{L}\right)$ is properly disjunctive iff for all sequence $\left(x_{i}\right)_{i \in \omega}$ of elements of $S$, the set $\operatorname{LIM}^{\mathrm{d}}\left(\mathrm{x}_{\mathrm{i}}\right)$ has the maximum that it is not $\mathbf{1}_{\mathrm{L}}$.

On one hand, if we consider classical propositional logic $\mathrm{C}$, it is easy to see that $\mathrm{C}$ is a conjunctive and disjunctive logic. But $\mathrm{C}$ is neither properly conjunctive nor properly disjunctive. For example, the sequence of all propositional variables has no conjunctive and disjunctive limits different from $\mathbf{1}_{\mathrm{L}}$ and $\mathbf{0}_{\mathrm{L}}$. On the other hand, if we consider infinitary classical propositional logic $\mathrm{C}_{\infty}$, with infinitary conjunctions and disjunctions, we have a properly conjunctive and disjunctive logic.

The concept of a finitely trivializable system is used to refer to a logic containing a formula from which everything (i.e. all formulas in the language) can be deduced (cf. [6]). We can say that if a logic is conjunctive (but not properly conjunctive), then it is finitely trivializable. In this sense, the system $C_{\omega}$ in da Costa's hierarchy is not a conjunctive logic while $C_{1}$ is a conjunctive and a disjunctive logic.

\section{Limits of Sequences and Modal Operators}

We have argued (with Hilan Bensusan) in [1] that logical possibility and logical necessity are never absolute in the precise sense that what is logically possible in a given logic could be logically impossible in a different logic and vice-versa. The same applies, then, for logical necessity and, in more general terms, for all logical truths. So, it is inside a given logic that something is logically possible or not. We take, then, logical possibility with respect to a given logic as the largest concept in such a way that all kinds of empirical possibility (weaker possibilities) are particular cases of it (let's call them X-possibilities for X being a particular theory, as suggested in [3]). In this way, if something is X-possible, then it is logically possible (in a formal system taken as underlying logic of a given theory). ${ }^{9}$ This obviously gives a clue to the fact that logical possibility is a kind of limit of a sequence of modal $\diamond$-formulas. ${ }^{10}$ Conversely, logical necessity can also be viewed as a sort of limit of a sequence of $\square$-formulas, considering that if something is a logical necessity, then it is an $\mathrm{X}$-necessity. So, for this reason, let us concentrate here in the case of modal operators, especially those of the form $\diamond$ and of the form $\square$.

Assume a family of normal modal logics with finitely many modal operators. Let $\mathrm{Y}_{1}, \ldots, \mathrm{Y}_{\mathrm{n}}$ be this multimodal system such that for each $Y_{i}$ there is a $\diamond_{i}$ and a respective definable $\square_{i}$. From the viewpoint of abstract logic, this system is a multimodal abstract logic $(\mathrm{S}, \mathrm{Cn})$ such that $\mathrm{S}$ contains sequences of modal operators $\left\{\diamond_{i}\right\}_{i \in \omega}$ and $\left\{\square_{i}\right\}_{i \in \omega}$. As mentioned, these operators could represent different kinds, degrees, levels of possibilities and necessities (X-possibilities and so on). Moreover, suppose that $(\mathrm{S}, \mathrm{Cn})$ is a properly conjunctive and a properly disjunctive logic.

Let $x$ be an element of $S$ and consider the sequence $\left\{\diamond_{i} x\right\}_{i \in \omega}$ of elements of $S$. In this way, we could define logical possibility $\diamond$ in $(\mathrm{S}, \mathrm{Cn})$ as a disjunctive limit of this sequence, that is:

$$
\diamond_{\mathrm{x}}:=\lim ^{\mathrm{d}}\left(\diamond_{\mathrm{i}} \mathrm{x}\right)=\max \left(\mathbf{L I M}^{\mathrm{d}}\left(\diamond_{\mathrm{i}} \mathrm{x}\right)\right) .
$$


Similarly, we could define the logical necessity $\square$ in (S,Cn) as a conjunctive limit of this sequence, that is:

$$
\square \mathrm{x}:=\lim ^{\mathrm{c}}\left(\square_{\mathrm{i}} \mathrm{x}\right)=\min \left(\mathbf{L I M}^{\mathrm{c}}\left(\square_{\mathrm{i}} \mathrm{x}\right)\right) .
$$

If we take logical possibility and logical necessity as above, we could say that multimodal systems with interactive axioms regulating levels of modal operators can be viewed as logics in which there are conjunctive and disjunctive limits. So, in the first case, we would have a logic (where $\otimes$ represents fusions of logics) $)^{11}$

$$
Y_{1} \otimes \ldots \otimes Y_{n} \otimes\left(\diamond_{1} a \rightarrow \diamond_{2} a\right) \otimes \ldots \otimes\left(\diamond_{n-1} a \rightarrow \diamond_{n} a\right)
$$

and logical possibility $\diamond_{\mathrm{a}}$ is $\diamond_{\mathrm{n}} \mathrm{a}$. The relevant fact is that all other kinds of possibility imply logical possibility in such a way that this one can be viewed, therefore, as a disjunctive limit. In the second case, conversely, we would have a logic

$$
\mathrm{Y}_{1} \otimes \ldots \otimes \mathrm{Y}_{\mathrm{n}} \otimes\left(\square_{\mathrm{n}} \mathrm{a} \rightarrow \square_{\mathrm{n}-1} \mathrm{a}\right) \otimes \ldots \otimes\left(\square_{2} \mathrm{a} \rightarrow \square_{1} \mathrm{a}\right)
$$

and logical necessity $\square \mathrm{a}$ is $\square_{\mathrm{n}}$ a. Now, the relevant fact is that logical necessity implies all kinds of necessity and, therefore, it is a conjunctive limit. So, in multimodal logics with ordered modal operators, it is very natural to think about conjunctive and disjunctive limits. Thus, we can say that logical possibility is the disjunctive limit of a sequence of weaker sorts of possibilities (as each Xpossibility implies logical possibility) and the dual logical necessity is the conjunctive limit of a sequence of stronger kinds of necessities (if something is a logical necessity, then it is X-necessary).

Considering that logical possibility (and its dual logical necessity) are always determined with respect to a given logic, it follows that hierarchies of weaker possibilities (and necessities) are also with respect to a given logic. Therefore, for each logical diamond or box, we have a respective hierarchy of X-possibilities (necessities) in a previous theory.

\section{Conclusion}

Limits of sequences of formulas (and, in particular, modal formulas) have a wide variety of applications. Treating logical possibility and logical necessity as disjunctive and conjunctive limits suggests that it is feasible to define other dual concepts in a similar fashion. The notion of disjunctive limit of a sequence involves the idea that the disjunctive limit can be derived from any element in the sequence, and it allows us to define the notion of disjunctive logic. The idea of conjunctive limit of a sequence accepts that a conjunctive limit implies any element of the sequence, and, as such, it can be used to define a conjunctive logic. The contributions of this paper are conceptual in the sense that definitions were designed to be applied in logical research. As in many situations we find hierarchies of sentences, limits can always be launched, and, therefore, definitions suggested here have a large scope of applications. It seems that these abstractions also facilitate attempts to model some situations in mathematics and philosophy.

\section{Acknowledgement}

This paper is dedicated to Jan Woleński for the celebration of his 80th birthday. Thanks to Andrew Schumann for the invitation to contribute with this special issue of Studia Humana. 


\section{References}

1. Bensusan, H., A. Costa-Leite, and E. G. de Souza. Logics and their galaxies, In A. Koslow and A. Buchsbaum (eds.), The Road to Universal Logic, vol. 2, Basel: Birkhäuser, 2015, pp. 243-252.

2. Beziau, J-Y. Recherches sur la logique universelle (excessivité, négation et séquents, $\mathrm{PhD}$ Thesis, Université Denis-Diderot Paris 7, 1995.

3. Costa-Leite, A. Logical properties of imagination. Abstracta 6 (1), 2010, pp. 103-116.

4. Costa-Leite, A., and E. G. de Souza. Implications and limits of sequences, Studia Humana 6 (1), 2017, pp. 18-24.

5. Costa-Leite, A. Paraconsistência, modalidades e cognoscibilidade, Available on PhilArchive: retrieved June 17, 2020 from https: //philarchive.org/archive/COSPME-5, 2019 (in Portuguese).

6. da Costa, N. On the theory of inconsistent formal systems, Notre Dame Journal of Formal Logic 15 (5), 1974, pp. 497-510.

7. Dunn, M, and G. Hardegree. Algebraic Methods in Philosophical Logic, New York: Oxford University Press, 2001.

8. Gabbay, D., A. Kurucz, F. Wolter, and M. Zakharyaschev. Many-dimensional modal logics: theory and applications, Studies in Logic and the Foundations of Mathematics, Amsterdam: Elsevier, 2003.

9. Tarski, A. On some fundamental concepts of metamathematics, In J. Corcoran (ed.), Logic, Semantic, Metamathematics, Second Edition, Indianapolis: Hackett Publishing Company, 1983.

10. Tarski, A. Fundamental concepts of the methodology of deductive sciences, In J. Corcoran (ed.), Logic, Semantic, Metamathematics, Second Edition, Indianapolis: Hackett Publishing Company, 1983.

\section{Notes}

1. These two concepts were initially proposed in [4], but without appeal to abstract logic.

2. A textbook relating ideas of algebra with logic in the domains of algebraic logic and algebra of logic can be found in [7].

3. We omit the subscript $\mathrm{L}$.

4. Other forms of logical consequence could be defined taking into account objects without making any reference to the linguistic dimension of $\mathrm{S}$.

5. Again, the subscript $\mathrm{L}$ is omitted.

6. This terminology is due to Jean-Yves Béziau in [2].

7. The subscript $\equiv$ is omitted.

8. In classical logic, a contradiction has this role, though it is not like this in all formal systems.

9. A hierarchy of diamonds have been used in [3] to build a combined logic of imagination, for instance.

10. A previous characterization of diamonds and boxes as limits of sequences of modal operators has been formerly developed in [5].

11. Cf. [8] for a roadmap with respect to combining logics in the environment of modal logics. 